\title{
Pain in relationship to Tinnitus and Hearing Loss
}

Henk M Koning ${ }^{1 *}$

Bas C. ter Meulen²

\begin{abstract}
Introduction: Cervical and lower back pain are noteworthy in the manner of development of tinnitus.

Objectives: The focus of this research was to indicate the consequence of the severity of neck pain and pain of the lower back and/ or lower limbs in tinnitus patients.

Design: A retrospective analysis of 61 patients with tinnitus as main complaint during a three month period.

Results: In this study, we found two groups of tinnitus patients defined by the existence of postural instability. Patients with tinnitus and postural unsteadiness were characterized by predominant female, self-perceived hearing loss, a higher intensity of tinnitus, cervical pain, and pain of the lower back and/or of the lower limbs, and more hearing deficit from $250 \mathrm{~Hz}$ to $4 \mathrm{kHz}$.

Conclusions: In patients with tinnitus one should be aware that hearing loss can be a consequence of high intensity cervical pain. Stimulation of the proprioceptive input pathways due to cervical pain can result in a higher intensity of tinnitus and a hearing loss in the range of $250 \mathrm{~Hz}$ to $4 \mathrm{kHz}$.
\end{abstract}

Keywords: Tinnitus; hearing loss; cervical pain; lower back pain; proprioception; dorsal column nuclei. 


\section{INTRODUCTION}

Tinnitus can be subdivided in two profiles of somatic tinnitus: tinnitus combined with postural unsteadiness and low-frequency hearing deprivation, and tinnitus with postural stability and no low-frequency hearing loss ${ }^{1}$. Pain may influence the sensitivity of the proprioceptive receptor leading to postural instability ${ }^{2}$. The intensity of cervical and lumbar pain is clearly related to the diminished equilibration ${ }^{2,3}$. Our hypothesis is that both cervical and lower back pain are noteworthy in the manner of development of tinnitus. Therefore, the focus of this research was to indicate the severity of neck pain and pain of the lower back and/or lower limbs in tinnitus patients.

\section{MATERIALS AND METHODS}

The Medical Ethics Committees United (Nieuwegein, the Netherlands) consented this observational retrospective research ${ }^{4}$ (W21.117, Mai 7, 2021).

Subjects: The totality of patients who paid a visit to our outpatient's department for tinnitus in a three months term $(1 / 1 / 2021-31 / 3 / 2021)$. All patients took counsel from an otorhinolaryngologist and all patients who had unilateral tinnitus underwent MRI to exclude pathology. Patients underwent an uniform clinical history and questionnaire of tinnitus, a two-sided audiogram, and a measurement of the intensity of tinnitus, neck pain, low back pain and/or pain of the lower limbs. The clinical history comprehended tinnitus features (left side and/or right side, traumatic origin, period of complaints and age of onset, and accompanying symptoms (self-reported hearing deficit, the presence of postural instability, dizziness, and neck pain). The Visual Analogue Scale (VAS) was used to measure the severity of pain and tinnitus.

Data Assessment: Features acquired from tinnitus patients were age, gender, severity of tinnitus or pain assessed by the VAS, together with the outcome of the tinnitus survey. The VAS is a 10-centimetre strip along the space separating the endings of "no tinnitus or pain" and "unbearable tinnitus or pain". The patient specified the smallest, the highest, and the average severity. The result was quantified by the interval (millimetre) between the "no tinnitus and pain" ending and the patient's token. A two-sided audiogram estimated tone limits at 250, 500, $1000,2000,4000$, and $8000 \mathrm{~Hz}$. The audiogram of the side with the most potent noise was appointed for the study. In case of two-side tinnitus with matching power, the average of both measurements was implemented.

Statistical Methods: Statistical calculation was performed with Minitab 18 (Minitab Inc., State College, PA, USA). Student's t-test was practised for continuous elements and $\mathrm{x} 2$ test for dichotomous elements. Discriminant analysis was tested to calculate the interrelationship of the severity of tinnitus or neck pain with the existence of postural instability and with hearing deficit at $250 \mathrm{~Hz}$ exceeding 23 decibel. A P-value smaller than 0.05 was significant.

\section{RESULTS}

From January 2021 to April 2021, 61 patients attended our clinic for their tinnitus. The features of these sufferers of tinnitus are demonstrated in Table 1. Tinnitus patients had a pervasiveness of postural instability, self-perceived hearing loss, and cervical pain.

Tinnitus patients with postural instability were set side by side with the patients with postural stability (Table 2). Patients with tinnitus and postural instability were characterised by predominant female, self-perceived hearing loss, a higher intensity of tinnitus, cervical pain, and pain of the lower back and/or of the lower limbs, more hearing deficit from $250 \mathrm{~Hz}$ to $4 \mathrm{kHz}$, and less sloping high frequency hearing deficit (i.e., ratio hearing loss at 8 $\mathrm{kHz}$ divided by hearing loss at $2 \mathrm{kHz}$ ).

Discriminant analysis for breaking up in two classes was performed to evaluate the association of the mean intensity of neck pain and pain of the lower back and/ or of the lower limbs with the existence of tinnitus with postural instability. The height of the mean intensity of cervical pain and of pain of the lower back and/or lower limbs could predict the appearance of postural instability in tinnitus patients (Proportion Correct of 0.667 and 0.750 , respectively). A VAS of the mean intensity of the cervical pain exceeding 31 millimetre was linked to an increased prevalence of postural unsteadiness from $29 \%$ to $62 \%$. Also, a VAS of the mean intensity of pain from lower back and/or lower limbs higher than 31 millimetres increased the commonness of postural unsteadiness from $23 \%$ to $72 \%$ in tinnitus patients.

In Table 3 patients with no mean pain exceeding 31 millimetres on the VAS were set side by side with patients suffering of only pain of the lower back and/or lower limbs, with patients with only neck pain, and with patients with neck pain together with pain of the lower back and/ or lower limbs, all with a mean pain intensity exceeding 31 millimetres on the VAS. Especially cervical pain with a mean intensity exceeding $31 \mathrm{~mm}$ on the VAS can induce hearing loss in the range between $250 \mathrm{~Hz}$ and $2 \mathrm{kHz}$. It seems that the presence of pain of the low back and/ or lower limbs with a mean pain intensity exceeding 31 millimetres on the VAS does not induce hearing loss itself but when it occurs together with cervical pain it had an additional effect in causing more hearing loss in the range between $250 \mathrm{~Hz}$ and $2 \mathrm{kHz}$. A VAS of the mean intensity of the cervical pain exceeding 31 millimetres has a sensitivity of $62 \%$ and a specificity of $79 \%$ for predicting the presence of tinnitus with postural instability. Positive and negative predictive values are $74 \%$ and $53 \%$ respectively.

Discriminant analysis for division in two classes was performed for the relationship of the ratio hearing loss at $8 \mathrm{kHz}$ divided by hearing loss at $2 \mathrm{kHz}$ with the existence of tinnitus without postural instability. The mean intensity of neck pain and of pain of the lower back and/or lower limbs could foretell the happening of postural stability in tinnitus patients (Proportion Correct of 0.600 ). 
Table 1. Clinical characteristics of the patients with tinnitus $(n=61)$.

\begin{tabular}{|c|c|c|c|}
\hline & Prevalence & \multicolumn{2}{|c|}{ Median- Q1-Q3 } \\
\hline Gender (male) & $56 \%$ & & \\
\hline Postural instability (yes) & $43 \%$ & & \\
\hline Self-perceived hearing loss (yes) & $67 \%$ & & \\
\hline Cervical pain (yes) & $72 \%$ & & \\
\hline Age at the start of the complaint (years) & & 51 & $36-59$ \\
\hline Perceived mean intensity of tinnitus ( $\mathrm{mm})$ : & & 72 & $50-85$ \\
\hline Perceived mean intensity of cervical pain (mm) & & 20 & $2-54$ \\
\hline Perceived mean intensity of pain of lower back and/or lower limbs (mm) & & 23 & $1-53$ \\
\hline
\end{tabular}

Q1 - Q3: inter-quartile range; mm: millimetre.

Table 2: Tinnitus patients with postural instability were compared with tinnitus patients without postural instability.

\begin{tabular}{|c|c|c|c|c|c|c|c|c|c|}
\hline & & \multicolumn{3}{|c|}{ Postural instability $(n=26)$} & \multicolumn{3}{|c|}{ Postural stability $(n=35)$} & \multicolumn{2}{|l|}{ P-value } \\
\hline \multirow{2}{*}{\multicolumn{2}{|c|}{ Gender (Male) }} & Prev. & Mean & SEM & Prev. & Mean & SEM & & \\
\hline & & $38 \%$ & & & $69 \%$ & & & 0.019 & S \\
\hline \multicolumn{2}{|c|}{ Self-perceived hearing loss (yes) } & $88 \%$ & & & $51 \%$ & & & 0.002 & $\mathrm{~S}$ \\
\hline \multicolumn{2}{|c|}{ Cervical pain (yes) } & $80 \%$ & & & $66 \%$ & & & 0.226 & \\
\hline \multicolumn{2}{|c|}{ Age at the start of the complaint (years) } & & 47 & 3.0 & & 48 & 2.5 & 0.973 & \\
\hline \multicolumn{2}{|c|}{ Perceived mean intensity of tinnitus (mm): } & & 73 & 5.1 & & 60 & 4.1 & 0.045 & $\mathrm{~S}$ \\
\hline \multicolumn{2}{|c|}{ Perceived mean intensity of cervical pain (mm) } & & 41 & 6.2 & & 22 & 4.8 & 0.020 & $\mathrm{~S}$ \\
\hline \multicolumn{2}{|c|}{$\begin{array}{l}\text { Perceived mean intensity of pain of the lower back and/or lower } \\
\qquad \text { limbs ( } \mathrm{mm})\end{array}$} & & 45 & 6.0 & & 18 & 4.0 & 0.001 & S \\
\hline \multicolumn{10}{|c|}{ Hearing loss $(\mathrm{dB})$ at: } \\
\hline- & $250 \mathrm{~Hz}$ & & 35 & 5.8 & & 16 & 2.8 & 0.005 & S \\
\hline- & $500 \mathrm{~Hz}$ & & 35 & 5.6 & & 16 & 2.7 & 0.004 & $\mathrm{~S}$ \\
\hline- & $1 \mathrm{kHz}$ & & 39 & 5.7 & & 18 & 2.9 & 0.002 & $S$ \\
\hline - & $2 \mathrm{kHz}$ & & 40 & 5.0 & & 20 & 2.6 & 0.001 & S \\
\hline- & $4 \mathrm{kHz}$ & & 50 & 5.1 & & 36 & 3.6 & 0.027 & $S$ \\
\hline - & $8 \mathrm{kHz}$ & & 60 & 5.3 & & 47 & 4.6 & 0.073 & \\
\hline \multicolumn{2}{|c|}{ Ratio hearing loss at $8 \mathrm{kHz}$ compared to hearing loss at $2 \mathrm{kHz}$} & & 1.9 & 0.2 & & 2.8 & 0.3 & 0.045 & $S$ \\
\hline
\end{tabular}

dB: decibel; Hz: Hertz; KHz: Kilohertz; Prev.: Prevalence; SEM: Standard Error of the Mean; mm: millimetre; S: Significant.

Table 3: The influence of the intensity of cervical pain and pain of the lower back and/or lower limbs on hearing loss in the range between $250 \mathrm{~Hz}$ and $8 \mathrm{kHz}$

\begin{tabular}{|c|c|c|c|c|c|c|}
\hline Mean VAS pain & $\begin{array}{c}\text { No pain }>31 \\
\mathrm{~mm}\end{array}$ & $\begin{array}{l}\text { Only pain low back and/or } \\
\text { lower limbs }>31 \mathrm{~mm}\end{array}$ & $\begin{array}{l}\text { Only cervical pain > } \\
\qquad 31 \mathrm{~mm}\end{array}$ & $\begin{array}{c}\text { Both cervical pain and pain } \\
\text { lower back and/or lower limbs } \\
>31 \mathrm{~mm}\end{array}$ & P-value & \\
\hline Hearing loss $(\mathrm{dB})$ at: & Mean SEM & Mean SEM & Mean SEM & Mean SEM & & \\
\hline $250 \mathrm{~Hz}$ & 162.7 & $14 \quad 6.4$ & 288.8 & 428.1 & 0.004 & $S$ \\
\hline $500 \mathrm{~Hz}$ & $15 \quad 2.9$ & $16 \quad 6.9$ & 278.1 & 407.6 & 0.006 & $S$ \\
\hline $1 \mathrm{kHz}$ & $16 \quad 2.9$ & $18 \quad 4.9$ & 329.1 & $46 \quad 7.7$ & 0.001 & S \\
\hline $2 \mathrm{kHz}$ & $22 \quad 3.3$ & $26 \quad 5.4$ & $26 \quad 6.4$ & $44 \quad 7.1$ & 0.011 & S \\
\hline $4 \mathrm{kHz}$ & 394.5 & $46 \quad 6.2$ & 297.4 & 516.9 & 0.149 & \\
\hline $8 \mathrm{kHz}$ & $51 \quad 5.9$ & 578.6 & 399.5 & $59 \quad 5.9$ & 0.398 & \\
\hline Mean VAS tinnitus & 565.1 & 727.3 & 675.3 & $77 \quad 5.8$ & 0.034 & $\mathrm{~S}$ \\
\hline
\end{tabular}

dB: decibel; Hz: Hertz; KHz: Kilohertz; SEM: Standard Error of the Mean; mm: millimetre; S: Significant; VAS: visual analogue scale.

A ratio hearing loss at $8 \mathrm{kHz}$ divided by hearing loss at $2 \mathrm{kHz}$ exceeding 2.34 was associated with an increased prevalence of tinnitus without postural instability from $47 \%$ to $74 \%$. Also, it has an affectability of $45 \%$ and an explicitness of $79 \%$ in anticipating tinnitus without postural instability. The positive and negative prescient qualities were $74 \%$ and $53 \%$ separately.

\section{DISCUSSION}

In this study, we found two groups of tinnitus patients defined by the existence of postural instability. Patients with tinnitus and postural unsteadiness were characterised by predominant female, self-perceived hearing loss, a higher intensity of tinnitus, cervical pain, and pain of the lower back and/or of the lower limbs, and more hearing deficit from $250 \mathrm{~Hz}$ to $4 \mathrm{kHz}$.

There is support for two profiles of somatic tinnitus, separated by the existence of postural unsteadiness and low-frequency hearing deficit (Figure 1) 1 . The concurrence of tinnitus with postural instability commence as a cervical pain syndrome and the severity of the neck pain is related to the happening of postural unsteadiness. Postural steadiness is based on intricate interconnections between the proprioceptive, visual and vestibular sensory systems. 


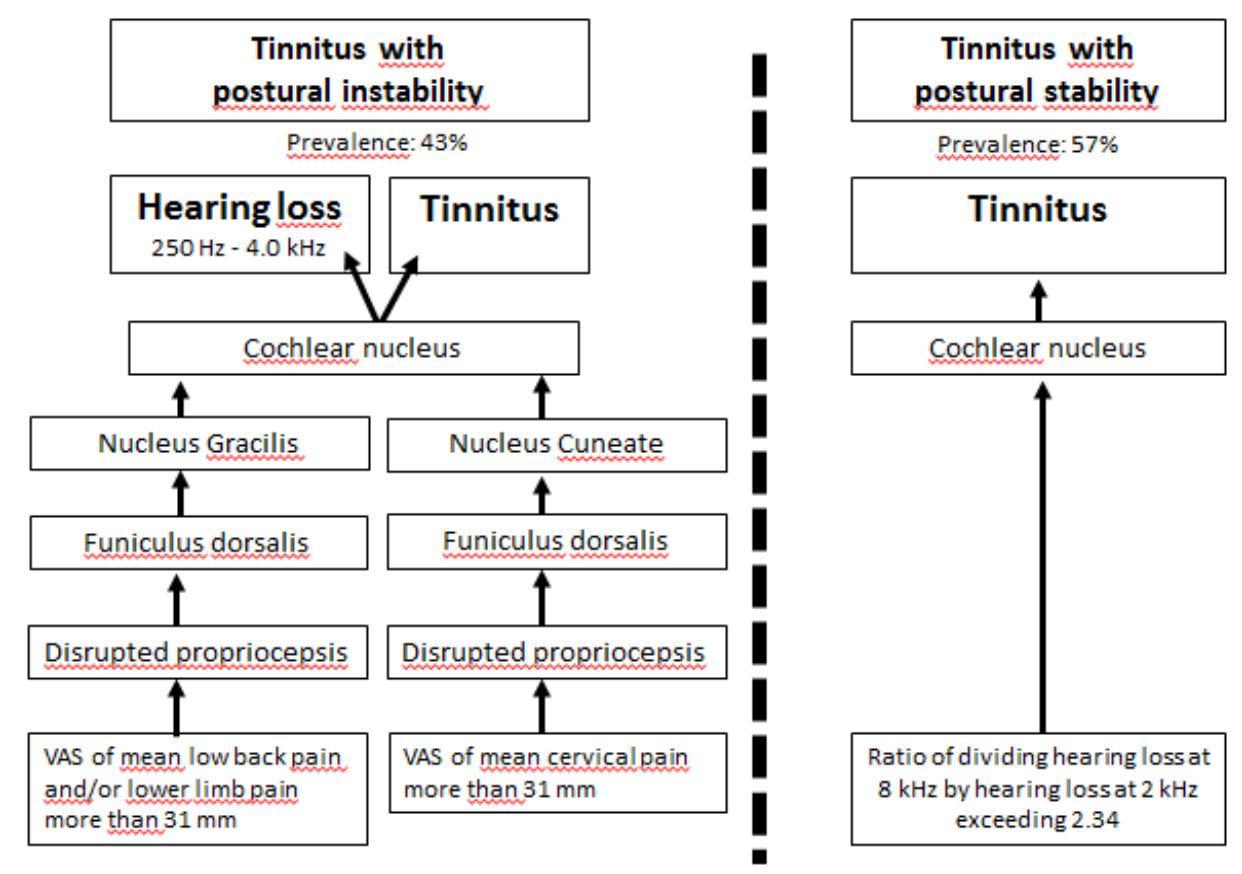

Figure 1: Theoretical model of the pathogenesis of tinnitus.

Proprioceptive information might be unsettled due to the activation of nociceptors. Pain intensity in patients with low back pain and with neck pain is compatible with a worsening degree of proprioception ${ }^{2,3}$. Also, in our study postural instability in tinnitus patients was related to high intensity pain of the neck, lower back and lower limbs ${ }^{4}$.

It is known that several chronic pain syndromes are linked with hearing loss ${ }^{5}$. Cervical spine and temporomandibular disorders may include hearing loss, vertigo, or tinnitus ${ }^{6,7}$. Patients with temporomandibular illness and patients with migraine have hearing deterioration at low frequencies ${ }^{8,9}$. Individuals with fibromyalgia and people with local and widespread musculoskeletal pain not diagnosed with fibromyalgia are also prone to subjective hearing loss ${ }^{10}$. Taken together, these findings point towards a direct link between the auditory and pain systems.

There are two discriminative somatosensory ways to the cochlear nucleus ${ }^{11,12}$. First, a fast pathway by mossy fibres from the dorsal column nuclei to the dorsal cochlear nucleus cells. Second, a slower pathway of the primary somatic ganglion cells with small bouton endings that activates both dorsal and ventral cochlear nucleus. The dorsal column-medial lemniscus system transport proprioceptive information from the dorsal root ganglion to the two dorsal column nuclei: the cuneate nucleus and the gracile nucleus. The cuneate nucleus collects its input from the upper part of the body, whereas the gracile nucleus obtains input from the lower part. Stimulation of the proprioceptive input pathways due to cervical pain can influence the intensity coding of acoustic impulses by auditory neurons resulting in hearing loss and a higher intensity of tinnitus ${ }^{11}$. This study demonstrates that the activation of the cuneate nucleus seems more important than the activation of the gracile nucleus in tinnitus patients resulting in a hearing deficit in the range of $250 \mathrm{~Hz}$ to $4 \mathrm{kHz}^{12}$. However, activation of the gracile nucleus seems to empower the influence of activation of the cuneate nucleus on hearing loss.

It has been stated that destruction of the acoustic input pathway provides raised excitatory somatosensory inputs to the cochlear nucleus ${ }^{13}$. This can result in hyperactivity in the dorsal cochlear nucleus and to an altered distribution and/or number of neurotransmitter receptors in the ventral cochlear nucleus ${ }^{13,14}$. However, the upregulation of glutamatergic neurotransmission can also be due to increased excitatory non-auditory projections to the cochlear nucleus ${ }^{15}$. Our study points out that the activation of non-auditory pathways to the cochlear nucleus can possibly cause hearing loss. Especially, if the proprioceptive nervous system is disrupted by high intensity cervical pain hearing loss can ensue. Therefore, in patients with tinnitus one should be aware that hearing loss can be a consequence of high intensity cervical pain.

In our study, a higher ratio of hearing loss at $8 \mathrm{kHz}$ divided by the hearing loss at $2 \mathrm{kHz}$ is associated with tinnitus without postural instability. An inductor of tinnitus may be a steep edge of hair cell loss ${ }^{16,17}$. Neurons in frequency regions not affected by hearing loss become hyperactive because of reduced inhibition from damaged neurons encoding neighbouring frequencies. A further proposed inductor of tinnitus is a mismatch between outer and inner hair cell loss at the same frequency ${ }^{16,18}$. Inhibitory inputs to the dorsal cochlear nucleus arises from outer hair cells. Larger damage of outer hair cell than inner hair cell at the border of the cochlear lesion impair inhibition more resulting in hyperactivity in the cochlear nucleus ${ }^{18}$.

Limitations of This Study. The population of people with tinnitus who visited our clinic may not be the prototypical 
example of the tinnitus community. Especially patients with high intensity tinnitus and severe psychosocial suffering can be overrepresented as they are in need for therapy. The use of VAS scales to objectivity the severity of tinnitus and pain can be a subject for debate. However, VAS scales are considered to be valid and reliable brief screening tools for tinnitus and pain ${ }^{19,20}$. Another confinement is its retrospective nature and prospective follow-up research is recommended to validate the results and the conclusions of this study.

\section{CONCLUSION}

There is support for two profiles of somatic tinnitus, separated by the existence of postural unsteadiness and low-frequency hearing deficit. A higher ratio of hearing loss at $8 \mathrm{kHz}$ divided by the hearing loss at $2 \mathrm{kHz}$ is linked to tinnitus with postural stability. Postural instability in tinnitus patients is associated with high intensity pain of the neck, lower back and lower limbs. Stimulation of the proprioceptive input pathways due to cervical pain is related to a higher intensity of tinnitus and a hearing loss in the range of $250 \mathrm{~Hz}$ to $4 \mathrm{kHz}$. Therefore, in patients with tinnitus one should be aware that hearing loss can be related to high intensity cervical pain.

\section{CONFLICT OF INTEREST}

The authors declares no conflict of interest

\section{REFERENCES}

1. Koning HM. Proprioception: the missing link in the pathogenesis of tinnitus? International Tinnitus J. 2020;24:102-7.

2. Reddy RS, Tedla JS, Dixit S, Abohashrh M. Cervical proprioception and its relationship with neck pain intensity in subjects with cervical spondylosis. BMC Musculoskelet Disord. 2019;20:447-54.

3. Roosink M, McFadyen BJ, Hébert LJ, Jackson PL, Bouyer LJ, Mercier C. Assessing the perception of trunk movements in military personnel with chronic non-specific low back pain using a virtual mirror. PLoS One. 2015;10:e0120251.

4. Peng B, Yang L, Li Y, Liu T, Liu Y. Cervical Proprioception Impairment in Neck Pain-Pathophysiology, Clinical Evaluation, and Management: A Narrative Review. Pain Ther. 2021;10:143-64.

5. Manohar S, Adler HJ, Radziwon K, Salvi R. Interaction of auditory and pain pathways: Effects of stimulus intensity, hearing loss and opioid signalling. Hear Res. 2020;393:108012-14.

6. Vasaghi-Gharamaleki B, Naser Z. Predicting the Risk of Hearing Impairment Following the Cervical Spine Diseases by Measuring the Cervical Range of Movements: A Pilot Study. Basic Clin Neurosci. 2017;8:413-8.
7. Maciel LFO, Landim FS, Vasconcelos BC. Otological findings and other symptoms related to temporomandibular disorders in young people. Br J Oral Maxillofac Surg. 2018;56:739-43.

8. Arakaki X, Galbraith G, Pikov V, Fonteh AN, Harrington MG. Altered brainstem auditory evoked potentials in a rat central sensitization model are similar to those in migraine. Brain Res. 2014;1563:110-21.

9. Pekkan G, Aksoy S, Hekimoglu C, Oghan F. Comparative audiometric evaluation of temporomandibular disorder patients with otological symptoms. J Craniomaxillofac Surg. 2010;38:231-4.

10. Stranden M, Solvin H, Fors EA, Getz L, Helvik AS. Are persons with fibromyalgia or other musculoskeletal pain more likely to report hearing loss? A HUNT study. BMC Musculoskelet Disord. 2016;17:477-8.

11. Dehmel S, Cui YL, SE Shore SE. Cross-modal interactions of auditory and somatic inputs in the brainstem and midbrain and their imbalance in tinnitus and deafness. $J$ Neurosci. 2012;32:1660-71.

12. Shore S, Zhou J, Koehler S. Neural mechanisms underlying somatic tinnitus. Prog Brain Res. 2007;166:107-23.

13. Dehmel S, Pradhan S, Koehler S, Bledsoe S, Shore S. Noise overexposure alters long-term somatosensory-auditory processing in the dorsal cochlear nucleus--possible basis for tinnitus-related hyperactivity? J Neurosci. 2012;32:1660-71.

14. Fang L, Fu Y, Zhang TY. Salicylate-Induced Hearing Loss Trigger Structural Synaptic Modifications in the Ventral Cochlear Nucleus of Rats via Medial Olivocochlear (MOC) Feedback Circuit. Neurochem Res. 2016;41:1343-53.

15. Shore SE, Wu C. Mechanisms of Noise-Induced Tinnitus: Insights from Cellular Studies. Neuron. 2019;103:8-20.

16. Roberts LE, Eggermont JJ, Caspary DM, Shore SE, Melcher JR, Kaltenbach JA. Ringing ears: the neuroscience of tinnitus. J Neurosci. 2010;30:14972-9.

17. Henry JA, Roberts LE, Caspary DM, Theodoroff SM, Salvi RJ. Underlying mechanisms of tinnitus: review and clinical implications. J Am Acad Audiol. 2014;25:5-22.

18. Shore SE, Koehler S, Oldakowski M, Hughes LF, Syed S. Dorsal cochlear nucleus responses to somatosensory stimulation are enhanced after noise-induced hearing loss. Eur $\mathrm{J}$ Neurosci. 2008;27:155-68.

19. Raj-Koziak D, Gos E, Swierniak W, Rajchel JJ, Karpiesz L, Niedzialek I, et al. Visual Analogue Scales as a Tool for Initial Assessment of Tinnitus Severity: Psychometric Evaluation in a Clinical Population. Audiol Neurootol. 2018;23:229-37.

20. Kersten $P$, White $P J$, Tennant $A$. Is the pain visual analogue scale linear and responsive to change? An exploration using Rasch analysis. PLoS One. 2014;12:e99485. 